\title{
LENS SPACES AND DEHN SURGERY
}

\author{
STEVEN A. BLEILER AND RICHARD A. LITHERLAND
}

(Communicated by Frederick R. Cohen)

\begin{abstract}
The question of when a lens space arises by Dehn surgery is discussed with a characterization given for satellite knots. The lens space $L(2,1)$, i.e. real projective 3 -space, is shown to be unobtainable by surgery on a symmetric knot.
\end{abstract}

The problem of when a lens space can be obtained by performing Dehn surgery on a knot in the 3-sphere has been of interest to topologists for some time. It is known that certain lens spaces can arise by Dehn surgery on torus knots (Moser [Mo]), certain pretzel knots (Fintushel-Stern [FS]), and certain nontrivial satellite knots (in fact, certain cables of torus knots, BaileyRolfsen [BR]).

In this note we show how some recent developments in 3-manifold theory shed more light on this problem. In Theorem 1 we use recent results of CullerGordon-Luecke-Shalen [CGLS], Gabai [Ga], Gordon [Go], and Scharlemann [S] to characterize how a lens space can be obtained by surgery on a (nontrivial) satellite knot. Theorem 1 was originally proven by $\mathrm{Wu}$ [Wu]. We present here a somewhat more concise proof, discovered independently, which makes deeper use of the critical theorems of Gabai and Gordon. Similar results have also been obtained by Wang $\left[\mathrm{Wa}_{1}\right]$ and Hempel $[\mathrm{H}]$. We then specialize to the question of when real projective 3-space, i.e. the lens space $L( \pm 2,1)$, can be obtained by Dehn surgery. Using results of Thompson [T] and Wang [ $\left.\mathrm{Wa}_{2}\right]$, we show in Theorem 2 that no surgery on a nontrivial symmetric knot yields this manifold.

Theorem 1. If nontrivial Dehn surgery on a satellite knot yields a manifold with cyclic fundamental group, then the knot is a cable of a torus knot and the knot and surgery coefficient are as in [Go, Theorem 7.5 (iii), $k=2$ ]. I.e. the knot is the $(2 p q \pm 1,2)$-cable on a $(p, q)$-torus knot, the surgery coefficient is $4 p q \pm 1$, and the resulting manifold is $L\left(4 p q \pm 1,4 q^{2}\right)$.

Corollary. If a lens space $L$ is obtained by Dehn surgery on a satellite knot then $\left|\pi_{1}(L)\right| \geq 23$.

Received by the editors May 20, 1988 and, in revised form, January 23, 1989.

1980 Mathematics Subject Classification (1985 Revision). Primary 57N10, 57M25.

The first author was supported in part by NSF Grant DMS-8602327.

The second author was supported in part by NSF Grant DMS-8705760. 
Proof of Theorem 1. Our notation will mostly follow that of [Go]. Let $K$ be a nontrivial knot in the 3-sphere $S^{3}$, let $J$ be a knot nontrivially contained in $S^{1} \times D^{2}$ with winding number $w \geq 0$, and let $J(K)$ be the satellite of $K$ determined by $J \subseteq S^{1} \times D^{2}$. Further let $(J ; r)$ be the manifold obtained from $S^{1} \times D^{2}$ by $r$-surgery on $J, r \neq 1 / 0$; and let $(J(K) ; r)$ be the manifold obtained from $S^{3}$ by $r$-surgery on $J(K)$. Since $J(K)$ is not a torus knot, Corollary 1 of [CGLS] implies that $(J(K) ; r)$ does not have cyclic fundamental group unless $r$ is an integer; we therefore consider only surgery with an integral coefficient $m$ (so that our notation agrees with that of [Go]).

We begin by reviewing what is known when $(J ; m)$ has compressible boundary.

(1) By the proof of [S, Corollary 5.2], $w \neq 0$.

(2) The manifold $(J ; m)$ is homeomorphic to $V \# M$ where $V \cong S^{1} \times D^{2}$ and $M$ is some closed 3-manifold. Denoting the g.c.d. of $w$ and $m$ by $(w, m)$, we have [by Go, Lemma 3.3] that $H_{1}(M) \cong \mathbf{Z}_{(w, m)}$, and a meridian of $V$ has slope $m / w^{2}$ relative to the original $S^{1} \times D^{2}$. Moreover, by the proof of [Go, Lemma 3.6], $w$ and $m /(w, m)$ are relatively prime. It follows that $m / w^{2}$ has denominator at least $w$, and is therefore not an integer unless $w=1$.

(3) By [Ga, Theorem 1.1], either $H_{1}(M) \neq 0$ (i.e. $\left.(w, m) \neq 1\right)$ or $(J ; m)$ is homeomorphic to $S^{1} \times D^{2}$ and $J$ is a 0 - or 1-bridge braid. If $w=1$, then we must have the second possibility; but this implies that $J$ is a core of $S^{1} \times D^{2}$. Hence $w \geq 2$.

Now suppose that $(J(K) ; m)$ has cyclic fundamental group for some integer $m$. Then $(J ; m)$ has compressible boundary and all of the above applies. It follows that $(J(K) ; m) \cong\left(K ; m / w^{2}\right) \# M$. Hence $\left(K ; m / w^{2}\right)$ has cyclic fundamental group, and since $m / w^{2}$ is not an integer, Corollary 1 of [CGLS] implies that $K$ is a torus knot. Hence the fundamental group of $\left(K ; m / w^{2}\right)$ is nontrivial, and so the fundamental group of $M$ must be trivial. By (3) above, this implies that $(J ; m)$ is homeomorphic to $S^{1} \times D^{2}$ and $J$ is a 0 - or 1-bridge braid. A 0-bridge braid is a cable, so it only remains to eliminate the possibility that $J$ is a 1-bridge braid.

Suppose that $J$ is a 1-bridge braid. In $\S 3$ of [Ga] there are associated to $J$ two integers, the braid width $b(1 \leq b \leq w-2)$ and $t(1 \leq t \leq w-1)$. By [Ga, Lemma 3.5] and the remarks just before [Ga, Definition 3.3], the surgery coefficient $m$ is equal to $\pm(w t+d)$ depending on the orientation convention, where $d$ is either $b$ or $b+1$. On the other hand, as $K$ is a torus knot and as $\pi_{1}\left(K ; m / w^{2}\right)$ is cyclic, [Go, Corollary 7.4] implies that $m$ is congruent to $\pm 1 \bmod w^{2}$. (Note that $w$ and $m$ are relatively prime since $H_{1}(M)=0$.) Hence $d$ is congruent to $\pm 1 \bmod w$. Now $b$ is equal to either $d$ or $d-1$ and $1 \leq b \leq w-2$, so either $b=1$ or $b=w-2$. Replacing $J$ by its mirror image replaces $b$ by $w-b-1$ (see the remark after [Ga, Lemma 3.4]), so it 
is enough to consider the case $b=1$. But this means that $J$ is a $(2,1)$-cable of a cable (see the remark after [Ga, Examples 3.8]), so that $J(K)$ is a cable of a cable of a torus knot. This contradicts [Go, Theorem 7.5].

Theorem 2. Real projective 3-space cannot be obtained by Dehn surgery on a nontrivial symmetric knot $K$ in the 3-sphere.

Proof of Theorem 2. Recall that a knot $K$ in $S^{3}$ is symmetric if it is invariant under the action on $S^{3}$ of some nontrivial finite group $G$. Without loss of generality we may take $G$ to be a cyclic group $\mathbf{Z}_{n}$. From Moser [Mo] we conclude that $K$ is not a torus knot. We then reduce to the strongly invertible case by appealing to Wang $\left[\mathrm{Wa}_{2}\right]$. He proves that if $K$ admits an action of a cyclic group $\mathbf{Z}_{n}$ and is not a torus knot, then if $n>2$ or if $n=2$ and the action is fixed point free, no nontrivial surgery on $K$ yields a lens space. He also shows that if $n=2$ and $K$ is disjoint from the fixed point set, then no nontrivial surgery can yield real projective 3 -space. The only symmetry left to consider is a strong inversion.

So suppose that surgery on some nontrivial strongly invertible knot $K$ gives $R P^{3}$. As before, we begin by recalling some general facts about surgery on strongly invertible knots. The strong inversion on $K$ extends to an involution on each of the manifolds $(K ; r)$ obtained from $S^{3}$ by performing $r$-fold surgery on $K$. For each $(K ; r)$ the quotient under this involution is the 3-sphere and hence each $(K ; r)$ double branch covers $S^{3}$. Moreover, the branch set of this covering can be obtained by removing a trivial tangle from the unknot (i.e. the image of the axis of the strong inversion) and replacing it in a manner determined by the surgery coefficient $r$. See, for example, Montesinos [M] or, for a more explicit construction, [B].

As $K$ is not a torus knot, we can apply the cyclic surgery theorem of [CGLS] to conclude that the surgery coefficient $r$ is at a distance 1 from the meridian of $K$. It follows that the removal and replacement of the trivial tangle corresponding to $r$ is in fact the attachment of a band to the unknot. The core of this band is the image of our original knot $K$ under the quotient map $(K ; 1 / 0) \rightarrow S^{3}$; again see $[\mathrm{M}]$ or $[\mathrm{B}]$.

By Hodgson and Rubenstein [HR], lens spaces uniquely double branch cover the 3-sphere with branch set the appropriate two-bridge knot or link. For real projective space this two-bridge link is the Hopf link. We conclude that obtaining $R P^{3}$ by surgery on $K$ corresponds in the quotient $S^{3}$ to obtaining the Hopf link by attaching a band to the unknot.

Now we apply a theorem of Thompson [T, Corollary 3] which states that there is a unique band which creates the unknot from the Hopf link, or, dually, that there is a unique band which creates the Hopf link from the unknot. It follows that we have one of the two pictures in Figure 1, and that our original knot $K$ is unknotted. 


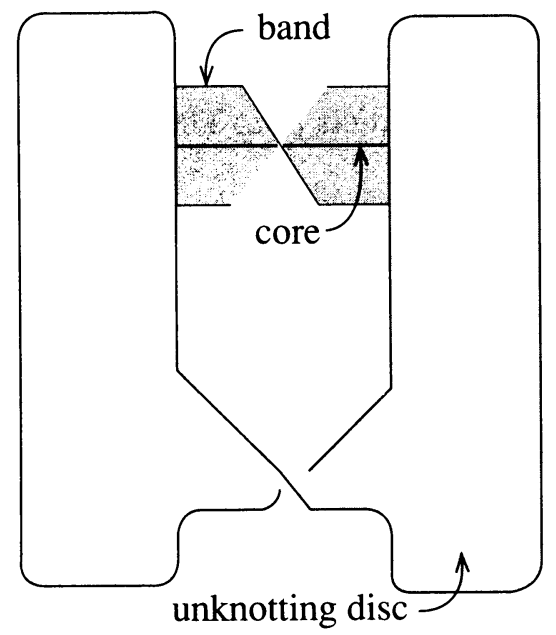

FIGURE 1 .

We close with some questions and conjectures.

(1) Is it possible to obtain a lens space $L$ with $\left|\pi_{1}(L)\right|<5$ by Dehn surgery on a nontrivial knot? Conjecture: No.

(2) It is possible to obtain a lens space $L$ with $\left|\pi_{1}(L)\right|<18$ by Dehn surgery on a nontorus knot? Conjecture: No.

\section{REFERENCES}

[BR] J. Bailey and D. Rolfsen, An unexpected surgery construction of a lens space, Pacific J. Math. 71 (1977), 295-298.

[B] S. A. Bleiler, Prime tangles and composite knots, Lecture Notes in Math. 1144, 1-13.

[CGLS] M. Culler, C. Gordon, J. Luecke, and P. Shalen, Dehn surgery on knots, Ann. of Math. (2), 125 (1987), 237-300.

[FS] R. Fintushel and R. Stern, Constructing lens spaces by surgery on knots, Math. Z. 175 (1980), 33-51.

[Ga] D. Gabai, Dehn surgery on knots in solid tori, preprint.

[Go] C. McA. Gordon, Dehn surgery and satellite knots, Trans. Amer. Math. Soc. 275 (1983), 687708.

[H] J. Hempel, Dehn fillings of coverings of surface bundles, Topology Appl. 24 (1986), 63-70.

[HR] C. Hodgson and H. Rubinstein, Involutions and isotopies of lens spaces, Lecture Notes in Math. 1144, 60-96.

[M] J. Montesinos, Surgery on links and double branched covers of $S^{3}$, Ann. of Math. Stud. 84 (1975) 227-260.

[Mo] L. Moser, Elementary surgery along torus knots, Pacific J. Math. 38 (1971), 734-745.

[S] M. Scharlemann, Sutured manifolds and generalised Thurston norms, preprint.

[T] A. Thompson, Unknotting number one knots are determined by their complements, preprint. 
[Wa $\left.\mathrm{W}_{1}\right] \mathrm{S}$. Wang, Cyclic surgery on knots, preprint.

$\left[\mathrm{Wa}_{2}\right] \ldots$, Symmetry of knots against cyclic surgery, preprint.

[Wu] $\mathrm{Wu}$, preprint.

Department of Mathematics, Portland State University, Portland, Oregon, 97207

Department of Mathematics, Louisiana State University, Baton Rouge, Louisiana 70803 